\title{
La enfermedad periodontal materna como factor de riesgo de prematuridad y bajo peso al nacer
}

The maternal periodontal disease as a risk factor of prematurity and low birth weight

\section{Resumen}

El objetivo del estudio fue evaluar la relación entre Enfermedad Periodontal (EP) materna con el parto prematuro (PP) y el bajo (BP) peso al nacimiento. El tipo de estudio es de casos y controles con 69 madres, 19 casos (madres de nacidos con PPBP) y 50 controles (madres de bebés con más de 37 semanas y más de 2500 g) en el Hospital Docente Madre Niño San Bartolomé. Se interrogó a las madres y se revisó las historias clínicas. Se registraron datos como: grado de instrucción, estado civil, hábitos de fumar, alcohol y drogas. Dentro de las 48 horas posteriores al parto, un solo examinador evaluó el índice de enfermedad periodontal (IEP) modificado registrando los indicadores periodontales: profundidad al sondaje, nivel de inserción clínica, sangrado al sondaje, inflamación y sangrado gingival y placa bacteriana. El análisis estadístico incluyó distribución de frecuencias y la prueba de Odds Ratio. Se encontró que las madres con peores condiciones de placa blanda OR: 1,45 (IC $95 \%$ 1,07-1,97); inflamación gingival OR: 2,14 (IC 95 \% 1,35- 3,39) y pérdida de nivel de inserción clínica OR: 2,8 (IC $95 \%$ $1,55-5,33)$ se asocian a nińos con PP y BP. La enfermedad periodontal materna incrementa el riesgo de prematuridad y bajo peso.

Palabras clave: Enfermedad periodontal, prematuro, recién nacido, peso neonatal.

\section{Abstract}

The objective of the study was to evaluate the relationship between the maternal periodontal disease (EP) and the premature delivery (PP) and the low birth weight (BP). The study was a case-control study with 69 mothers, 19 cases (mothers of newborns with premature deliveries and low birth weight (PPBP)), and 50 controls (mothers of babies with gestational age $>37$ weeks and birth weight $>2500 \mathrm{~g}$ ) at the "Madre Niño San Bartolomé" Teaching Hospital. The mothers were interrogated and their clinical histories reviewed. Data was recorded as follows: school level, marital status, smoking, alcohol and drug habits. Within 48 hours after birth delivery, one single examiner evaluated the modified periodontal disease index (IEP) recording the periodontal indicators: probing depth, clinical attachment level, bleeding on probing, gingival inflammation and bleeding and bacterial plaque. The statistical analysis included the frequency distribution and the Odds Ratio test. Mothers with worse bacterial plaque OR conditions: 1,45 (IC 95\% 1,07 - 1,97); gingival inflammation OR: 2,14 (IC 95\% 1,35 - 3,39) and clinical attachment level OR: 2,8 (IC 95\% 1,55 - 5,33) were found to be related to premature delivery and low-birth-weight children. The maternal periodontal disease increases the risk of premature births and low birth weight.

Key words: Periodontal disease, premature, newborn, neonatal weight.

\section{Introducción}

Los infantes con partos prematuros y bajo peso al nacer (PPBP) tienen 40 veces más probabilidades de morir en la etapa neonatal que los niños de peso normal. ${ }^{1}$ En nuestro país la mortalidad neonatal es de 10 por mil nacidos vivos según la Encuesta demográfica y de salud familia (ENDES 2007-2008), la tasa de mortalidad perinatal es de 19 defunciones por cada mil embarazos de 7 o más meses de duración con un componente mayor de nacidos muertos que de muertes tempranas; por otro lado, hay una tasa de $11 \%$ de bajo peso al nacer. $^{2}$
La identificación de los factores de riesgo para parto prematuro (PP) y bajo peso (BP) al nacer tiene un especial interés en salud pública; ya que, se asocia a mayor riesgo de morbimortalidad precoz, alteraciones congénitas e inmunológicas, y déficit del desarrollo neurológico. ${ }^{3}$ Además, son indicadores de malnutrición, falta de en atención en salud y pobreza. ${ }^{4}$

Existen factores que se han asociado a prematuridad como ruptura prematura de membranas e infecciones urinarias, sin embargo, el tratamiento de esta última ha reducido significativamente los partos prematuros., ${ }^{5,6}$ Algunos autores
Artículo Original

\section{Julissa Dulanto Vargas, ${ }^{1}$ Sixto García Linares, ${ }^{2}$ Maynor Carranza Samanez ${ }^{1}$}

\author{
${ }^{1}$ Práctica privada. \\ ${ }^{2}$ Docente del Dpto. Académico Médico Quirúr- \\ gico Facultad de Odontología de la UNMSM.
}

Correspondencia:

C.D. Julissa Amparo Dulanto Vargas

Av. Sucre 416, Lima 17. Perú

Teléfono: 989-035338

Correo electrónico: yuduva@hotmail.com
Fecha de recepción: 23-04-10

Fecha de aprobación: 08-11-10 clasifican los factores como: sociodemográficos, riesgos médicos anteriores y del embarazo actual, controles prenatales, riesgos ambientales y hábitos tóxicos. ${ }^{7,8}$ También existen estudios que mencionan la relación de madres de nacidos con PP y BP al nacer con una pobre salud periodontal. ${ }^{9-18}$

La infección es un factor clave para el embarazo adverso, tal es así, que la infección periodontal crónica puede provocar una respuesta local o sistémica que lleva a una bacteremia local o sistémica. Lipopolisacáridos, endotoxinas y otras sustancias bacterianas pueden invadir el tejido gingival activando la 
respuesta inflamatoria local y consecutivamente la producción de citoquinas proinflamatorias que entran en la circulación sanguínea materna afectando al feto. Esta activación en las gestantes es un factor patofisiológico que resulta en parto prematuro, bajo peso y preclampsia. ${ }^{19-21}$

En base a lo anterior, se requiere estudiar los factores influyentes para disminuir la elevada incidencia de mortalidad y morbilidad de nacidos prematuros y con bajo peso. Esto permitirá prevenir a tiempo y evitar el riesgo en los nińos.

El objetivo del estudio fue determinar la relación de la enfermedad periodontal materna, como factor de riesgo de parto prematuro y bajo peso en el Hospital Docente Madre Niño San Bartolomé (HODOMANI).

\section{Materiales y Método}

Estudio con diseño de casos y controles; la población estuvo conformada por madres que tuvieron su parto en el Departamento de Gineco-Obstetricia del Hospital Docente Madre Niño San Bartolomé (HODOMANI) durante los meses de julio a agosto de 2008. Se seleccionó a madres de recién nacidos vivos únicos que aceptaron participar en el estudio y que hubiesen llenado la hoja del consentimiento informado, con edades entre 20 y 35 ańos, sin enfermedad sistémica, $\geq 18$ piezas dentales, talla $\geq 1,5 \mathrm{~m}$, estado nutricional adecuado, más de una gestación y que no recibieron tratamiento antibiótica durante el último mes de gestación. La muestra estuvo conformada por 19 casos (recién nacidos con PPBP) y 50 controles (recién nacidos con más de 37 semanas de gestación y más de 2500 $\mathrm{g}$ de peso), la variable dependiente fue: Parto prematuro con bajo peso (PPBP).

El sistema informativo perinatal permitió evaluar los diagnósticos de parto prematuro y bajo peso al de los nacidos. $^{22}$

La variable independiente enfermedad periodontal, fue evaluada mediante el índice de enfermedad periodontal (IEP), ${ }^{23}$ modificado que considera los siguientes indicadores periodontales: profundidad al sondaje, nivel de inserción clínica, sangrado al sondaje, inflamación y sangrado gingival y placa.

Se evaluaron seis piezas dentarias: 16 , 21, 24, 36, 41 y 44; iniciando por el cuadrante superior derecha y finalizando el cuadrante inferior del mismo lado. Se evaluó la profundidad al son- daje y el nivel de inserción clínica utilizando una sonda calibrada $(\mathrm{mm})$ en 2 zonas del diente: vestibular y palatino. Se valoró los indicadores según los criterios del IEP: 4 = nivel de inserción $\leq$ $3 \mathrm{~mm}, 5=$ nivel de inserción $>3$ hasta $6 \mathrm{~mm}$ hacia apical, y $6=$ nivel de inserción $>6 \mathrm{~mm}$.

La evaluación de la inflamación, enrojecimiento y sangrado para gingivitis se realizó por vestibular (mesial, medio y distal) y por palatino. Los criterios para gingivitis fueron: 0 = ausencia, $1=$ leve, $2=$ moderada y 3 = severa).

La presencia de placa blanda se observó en las superficies vestibulares y palatinas y consideró el recubrimiento por superficie: ausencia, menos de $1 / 3$, hasta $2 / 3$, más de $2 / 3$ de la superficie evaluada.

Un encuestador recolectó la información demográfica (edad, grado de instrucción, estado civil), antecedentes médicos y atención obstétrica.

En el proceso: se revisaron las historias clínicas, luego se procedió a la explicación y llenado de la hoja de consentimiento. El examen clínico fue realizado por un examinador y transcrito a las fichas de recolección de datos, con las madres sentadas en su cama en el hospital e iluminación de una lámpara frontal. Para la evaluación periodontal se usó una sonda periodontal Hu Friedy (PCP 15), la evaluación se realizó dentro de las 48 primeras horas post parto.

Para el plan de análisis se realizó la verificación del ingreso de datos y análisis de rangos para determinar errores. El análisis estadístico consistió en el análisis univariado de frecuencias y porcentajes, o medias para cada una de las variables según casos y controles. Luego, se procedió al análisis bivariado de la relación de enfermedad periodontal y parto prematuro y bajo peso con el Odds Ratio (OR).

\section{Resultados}

La muestra efectiva del presente estudio estuvo conformada por 69 madres. Los rangos de edad para los casos fueron 26,4 ańos y para los controles 25,2 años.

En la Tabla 1 se muestra la distribución poblacional según características socioeconómicas. No hubo diferencias significativas en cuanto a grupo etáreo, grado de instrucción o estado civil ( $p>0,05)$. Ninguna mujer declaró hábitos de fumar, alcohol o drogas.

En la tabla 2, se muestra las comparaciones entre casos y controles según índice de enfermedad periodontal modificado. Los criterios de placa blanda, gingival y periodontal mostraron diferencias significativas, se observó tendencia de los casos a una peor condición periodontal que los controles.

En la tabla 3 se observa la asociación de PPBP con los criterios de placa blanda, gingival y periodontal del IEP modificado, muestra que las madres con peores condiciones periodontales tienen mayor probabilidad de tener nińos con PPBP.

\section{Discusión}

Actualmente se asocia la enfermedad periodontal con resultados desfavorables en la gestación. ${ }^{9-18}$ Estos efectos se han centrado en el término del embarazo (partos prematuros) y en el peso del nacido (bajo peso al nacer), más en aún, cuando se presentan ambas situaciones.

Tabla 1. Distribución de la muestra de estudio según características socioeconómicas, Hospital Docente Madre Niño San Bartolomé, 2008.

\begin{tabular}{lccc}
\hline VARIABLE & $\begin{array}{c}\text { CASOS } \\
(\mathbf{n}=\mathbf{1 9}) \%\end{array}$ & $\begin{array}{c}\text { CONTROLES } \\
(\mathbf{n}=\mathbf{5 0}) \%\end{array}$ & $\begin{array}{c}\text { Valor de } \\
\mathbf{p}\end{array}$ \\
\hline $\begin{array}{l}\text { Media de la edad materna } \\
\text { Estado civil }\end{array}$ & 26,4 & 25,2 & $>0,05$ \\
$\quad$ Soltera & 21 & & \\
Casada & 37 & 30 & $>0,05$ \\
Conviviente & 42 & 20 & \\
Grado de instrucción & & 50 & \\
$\quad$ Primaria & 36 & & $>0,05$ \\
Secundaria & 58 & 30 & \\
Superior & 6 & 62 & \\
\hline
\end{tabular}


Aún no hay consenso de definición exacta de EP ${ }^{24}$, requisito indispensable para un criterio uniforme global; sin embargo, la evaluación periodontal se basó en 2 de los parámetros más comunes usados para caracterizar a un paciente con periodontitis: NIC (considerado como el "gold Standard" de la medida de la destrucción periodontal) y PS, inflamación gingival, sangrado al sondaje y placa, ${ }^{25}$ que son evaluados mediante el IEP.

Existen factores de confusión para parto prematuro y bajo peso, la edad materna es una de ellas; sin embargo, el grupo de estudio fue bastante homogéneo con edades comprendidas entre 20 y 35 años de edad, con promedio para los casos de 26,4 y los controles de 25,2 ;

Tabla 2. Índice de enfermedad periodontal modificado, Hospital Docente Madre Niño San Bartolomé, 2008.

\begin{tabular}{lccc}
\hline VARIABLE & $\begin{array}{c}\text { CASOS } \\
(\mathbf{n = 1 9 ) \%}\end{array}$ & $\begin{array}{c}\text { CONTROL } \\
(\mathbf{n}=\mathbf{5 0} \%\end{array}$ & Valor de $\mathbf{p}$ \\
\hline $\begin{array}{l}\text { Criterio de placa blanda } \\
\text { Hasta 2/3 de placa }\end{array}$ & 15,8 & 42 & $<0,05$ \\
Más de 2/3 de placa & 84,2 & 58 & \\
\hline $\begin{array}{l}\text { Criterio gingival } \\
\text { Gingivitis ausente y leve }\end{array}$ & 21,1 & 78 & $<0,05$ \\
$\begin{array}{l}\text { Gingivitis moderada y } \\
\text { severa }\end{array}$ & 78,9 & 22 & \\
$\begin{array}{l}\text { Criterio periodontal } \\
\text { Nivel de inserción }\end{array}$ & 21,1 & 86 & \\
3mm & & & \\
$\quad$ Nivel de inserción $>$ & 78,9 & 14 & \\
3mm & & & \\
\hline
\end{tabular}

rangos similares al estudio de Radnai ${ }^{14}$ (edad promedio: casos 28,3 y controles 27,6). Estos rangos de edades no son de riesgo (edades inferiores a los 19-20 y mayores de 35-36 años), ${ }^{26}$ por tanto, no podría ser un factor influyente, además, no se encontró en asociación con PPBP.

El modelo de estudio excluyó las madres con hábitos de alcohol, cigarrillos y drogas, considerados como factores de riesgo de PP y BP según los estudios de Dasanayake $^{13}$ y Voigt ${ }^{26}$.

Tabla 3. Riesgo de índice de enfermedad periodontal modificado, Hospital Docente Madre Niño San Bartolomé, 2008.

\begin{tabular}{|c|c|c|c|c|c|}
\hline VARIABLE & CASOS & CONTROL & $\mathbf{p}$ & Riesgo & Rango \\
\hline Más de $2 / 3$ de placa & & & 0.041 & 1,45 & $1,07-1,97$ \\
\hline $\begin{array}{l}\text { Gingivitis moderada y } \\
\text { severa }\end{array}$ & $(n=19)$ & $(n=50)$ & 0.00 & 2,14 & $1,35-3,39$ \\
\hline $\begin{array}{l}\text { Nivel de inserción > } \\
3 \mathrm{~mm}\end{array}$ & & & 0.00 & 2,8 & $1,55-5,33$ \\
\hline
\end{tabular}

ORa (4,5 IC $95 \%$ 2,16-9,18); la EP y BP como Dasayanake ${ }^{13}$ [ORa 1,1 (0,97-1,40); finalmente, Offenbacher 17 [ORa $1,23(0,89-1,70)$ y ORa 2,12 $(1,34-3,35)]$ y Jarjoura ${ }^{18}$ [ORa 2,75 $(1,01-7,54)$ y ORa $1,99(0,73-5,45)]$ que asociaron la EP con PP y BP, respectivamente.

Por otra parte, difiere de algunos estudios que no han encontrado relación. Es posible que la discrepancia sea por diferencias en la definición de EP, así
Todas las madres que ingresaron al estudio provinieron de la misma fuente potencial de sujetos elegibles, madres que dieron a luz en el Hospital Docente Madre Niño San Bartolomé.

La evaluación de los criterios de IEP mostró diferencias estadísticas significativas $(\mathrm{p}<0.05)$ entre los grupos, con mayor tendencia de las madres de nińos con PPBP a mayor presencia de placa, inflamación y pérdida del nivel de inserción clínica (OR 2,8 IC95 \% 1,555,33). También se encontró riesgo en los estudios que asocian la EP y PPBP: Offenbacher' ${ }^{9}$ ORa 7,5 (1,95-28,8); Dörtbudak ${ }^{11}$ OR 20,0 (2,0-201,7); López $^{12}$ RRa 3,5 (1,5-7,9); Radnai ${ }^{14}$ OR 5,46 $(1,72-17,3)$ y Mokeem ${ }^{15}$ ORa 4,21 (1,99-8,93). Asimismo, otros que

como definición de los casos, ya que en algunos estudios consideran los componentes del PPBP por separado.

Algunos estudios que no encontraron relación son: Davenport ${ }^{27}$ ORa 0,78 $(0,64-0,99)$ con similares resultados de EP entre casos y controles en referencia a PP; Moore ${ }^{28}$ (EP en casos 2 $\%$ y control $4 \% \mathrm{p}=0,0016)$ no halló asociación de pérdida de inserción clínica $\geq 5$ mm y BP; Noack ${ }^{29}$ OR 0,191 (0,016-2,28) con resultados no significativos para la relación de PPBP y EP; Bassani $^{30}$ [OR 0,93 (0,63-1,41) y OR $0,92(0,54-1,57)]$ para la relación de EP con BP y PP, respectivamente; $\mathrm{Bu}-$ duneli ${ }^{31}$ (EP en casos 7,6 \% y control $9 \% \mathrm{p}=0,445)$ no encontró asociación de profundidad al sondaje $\geq 4 \mathrm{~mm}$ con respecto a madres de niños con PPBP. Davenport ${ }^{27}$ probablemente por el índice periodontal comunitario (CPITN) que usó, Moore ${ }^{28}$, probablemente, por el momento en el que realizó la evaluación periodontal a las $10-15$ semanas de embarazo; Noack ${ }^{29}$ por la definición de madres con EP, ( $>60 \%$ de sitios con NIC $>3 \mathrm{~mm}$ ) reduciendo la posibilidad de la madre de ser diagnosticada con EP.

\section{Conclusión}

La enfermedad periodontal materna es un factor de riesgo para el parto prematuro con bajo peso.

Las gestantes que presenten pérdida de inserción clínica $>3 \mathrm{~mm}$ en el periodonto tienen casi 3 veces mayor riesgo de dar luz nińos prematuros con bajo peso. OR 2,8 (IC9 5\% 1,55 - 5,33)

Las gestantes que presenten inflamación gingival moderada y severa tienen el doble de riesgo de dar luz niños prematuros con bajo peso. OR 2,14 (IC95 $\% 1,35-3,39)$

Las gestantes que presenten más de $2 / 3$ de placa bacteriana tienen un poco más de riesgo de dar luz niños prematuros con bajo peso. OR 1,45 (IC95 \% 1,07 $-1,97)$

Se recomienda prevenir y controlar la EP en gestantes para disminuir los PPBP. 


\section{Referencias bibliográficas}

1. McGaw T. Periodontal disease and preterm delivery of low-birthweight infants. J Can Dent Assoc 2002;68:165-69.

2. Instituto Nacional de Estadística e Informática. Encuesta demográfica y de salud familiar 2007-2008. [citado 20 abril de 2010]; Disponible en: http://desa.inei.gob.pe/ endes/endes 2007/Publicación\%20 ENDES.html

3. Cunninham G, McDonald PC, Gant N, Levenok, Gilstrop L. Embarazo ectópico. En: Williams Obstetricia. México: Masson; 1996.p.839-56.

4. Klebanov PK, Brooks-Gunn J, McCarton C, McCormick MC. The contribution of neighborhood and family income to developmental test scores over the first three years of life. Child development 1998;69:1420-36.

5. Díaz LM, Dinsmoor MJ, Lin PY. Preventable risk factors for the delivery of very low birth weigth infants in Richmond, Virginia. Prim Care Update Obstet Gynecol 2001;8:14.

6. Clausson B, Cuattinguis S, Axelsson O. Preterm and term births of small for gestational age infants: a population based study of risk factors among nulliparous women. $\mathrm{Br}$ J Obstet Gynecol 1998;105:10117.

7. Soriano T, Juarrauz M, Valero J, Martínez D, Calle M, Domínguez $\mathrm{V}$. Principales factores de riesgo del bajo peso al nacer. Análisis multivariante. Rev SEMG 2003;53:26370.

8. Bortman M. Factores de riesgo de bajo peso al nacer. Rev Panam Salud Pública 1998;3:314-21.

9. Offenbacher S, Katz V, Fertik G, Collins J, Boyd D, Maynor G, McKaig R, Beck J. Periodontal infection as a possible risk factor for preterm low birth weight. J Periodontol 1996;67:1103-07.

10. Offenbacher S, Jared HL, O'Reilly PG, Wells SR, Salvi GE, Lawrence HP, Socransky SS, Beck J. Potential pathogenic mechanisms of periodontitis - Associated pregnancy complications. Ann Periodontol 1998;3:233-48.

11. Dörtbudak O, Eberhardt MU, Persson UM. Periodontitis, a marker of risk pregnancy for preterm birth. J Clin Periodontol 2005;32:45-52.

12. Lopez NJ, Smith PC, Gutierrez J. Higher risk of preterm birth and low birth weight in women with periodontal disease. J Dent Res 2002;81:58-63.

13. Dasanayake AP. Poor periodontal health of the pregnant woman as a risk factor for low birth weight. Ann Periodontol 1998;3:206-12.

14. Radnai M, Gorzo I, Nagy E, Urban E, Novak T, Pal A. A possible association between preterm birth and early periodontitis. A pilot study. J Clin Periodontol 2004;31:736-41.

15. Mokeem SA, Molla GN, Al-Jewair TS. The prevalence and relationship between periodontal disease and pre-term low birth weight infants at King Khalid University Hospital in Riyadh, Saudi Arabia. J Contemp Dent Pract 2004;5:40-56.

16. Jeffcoat MK, Geurs NC, Reddy MS, Cliver SP, Goldenerg RL, Hauth JC. Periodontal infection and preterm birth: results of a prospective study. J Am Dent Assoc 2001;132:875-80.

17. Offenbacher S, Lieff S, Boggess KA, Murtha AP, Madianos PN, Champagne $\mathrm{CM}$, et al.Maternal periodontitis and prematurity. Part I: Obstetric outcome of prematurity and growth restriction. Ann Periodontol 2001;6:164-74

18. Jarjoura K, Devine PC, Perez-Delboy A, Herrera-Abreu M, D'Alton M, Papapanou PN. Markers of periodontal infection and preterm birth. Am J Obstet Gynecol 2005;192:513-19.

19. Garcia RI, Henshaw MM, Krall EA. Relationship between periodontal disease and systemic health. Periodontol 2000 2001;25:21-36.

20. Von Dadelszen P, Magee LA. Could an infectious trigger explain the differential maternal response to the shared placental pathology of preeclampsia and normotensive intrauterine growth restriction? Acta Obstet Gynecol Scand 2002;81:642-8.
21. Xiong X, Buekens P, Fraser WD, Beck J, Offenbacher S. Periodontal disease and adverse pregnancy outcomes: a systematic review. BJOG 2006;113:135-43.

22. Centro Informático de Perinatología Salud de la mujer y Reproductiva. Historia clínica perinatal. CLAP/SMR-OPS/OMS. 2007.

23. Ramfjord S. Indices for prevalences and incidence of periodontal disease. J Periodontol 1959; 30: 51-9.

24. Leroy R, Eaton KA, Savage A. Methodological issues in epidemiological studies of periodontitis - How can it be improved? BMC Oral Health 2010;10:8.

25. Lizarraga CA, Proaño D. La enfermedad periodontal como factor de riesgo de parto pretérmino y de bajo peso al nacer en el Hospital Nacional Cayetano Heredia 2002-2003. Rev Med Hered 2005;16:172-7.

26. Voigt M, Briese V, Pietzner V, Kirchengast S, Schneider KT, Straube $S$, Jorch G. Evaluation of maternal parameters as risk factors for premature birth (individual and combined effects). Z Geburtshilfe Neonatol 2009;213:138-46.

27. Davenport ES, Williams CE, Sterne JA, Murad S, Sivapathasundram V, Curtis MA. Maternal periodontal disease and preterm low birthweight: case-control study. J Dent Res 2002;81:313-18.

28. Moore S, Randhawa MS, Ide M. A case-control study to investigate an association between adverse pregnancy outcome and periodontal disease. J Clin Periodontol 2005;32:1-5.

29. Noack B, Klingenberg J, Weigelt J, Hoffmann T. Periodontal status and preterm low birth weight: a case control study. J Periodontal Res 2005;40:339-45.

30. Bassani DG, Olinto MTA, Kreiger N. Periodontal disease and perinatal outcomes: a casecontrol study. J Clin Periodontol 2007;34:31-9.

31. Buduneli N, Baylas H, Buduneli E, Türkoğlu O, Köse T, Dahlen G: Periodontal infections and pre-term low birth weight: a casecontrol study. J Clin Periodontol 2005;32:174-81. 